\title{
Simultaneous Quantification of Amorphous and Crystalline Valsartan in Tablets Using Raman Spectroscopy and Chemometrics Tools
}

\author{
Rafael C. Domingos, ${ }^{a}$ Adonilson F. Pinheiro, ${ }^{a}$ Benedito R. de Alvarenga Jr., ${ }^{b}$ \\ Renato L. Carneiro ${ }^{b}$ and Marco A. S. Farias ${ }^{\circledR} * a$ \\ ${ }^{a}$ Departamento de Química, Instituto de Ciências Exatas, Universidade Federal do Amazonas, \\ Avenida Gen. Rodrigo Octávio, 6200, Campus Universitário, Setor Norte, 69080-900 Manaus-AM, Brazil \\ ${ }^{b}$ Departamento de Química, Centro de Ciências Exatas e de Tecnologia, Universidade Federal de São Carlos, \\ Rodovia Washington Luis, Km 235, 13565-905 São Carlos-SP, Brazil
}

\begin{abstract}
Valsartan is an antihypertensive active pharmaceutical ingredient (API), it is used in the amorphous state in the commercial products. As amorphous materials are metastable, amorphous valsartan can crystallize to valsartan E, promoting changes in the dissolution and bioavailability of the drug. Tablets containing metastable forms of APIs need special conditions for transport and storage in order to avoid crystallization (from amorphous state) or polymorphic transitions (from less stable crystalline structures). A multivariate calibration model based on interval partial least squares (iPLS) regression allied to net analyte signal (NAS) algorithm was built to simultaneously quantify amorphous (VAL-AM) and crystalline (VAL-E) valsartan. Mixtures of VAL-AM and VAL-E were used to produce tablets in order to simulate the crystallization of VAL-AM in a range from 0 to $100 \%$ of conversion. The calibration set included 11 samples and 5 samples were used as the external validation set. The following parameters of merit (POM) were obtained for both polymorphs in order to evaluate the calibration model quality: root mean square error (RMSE) for cross validation (RMSECV), validation (RMSEV) and calibration (RMSEC), sensitivity (SEN), selectivity (SEL), analytical sensitivity $(\gamma)$, inverse analytical sensitivity $\left(\gamma^{-1}\right)$, limit of detection (LOD) and limit of quantification (LOQ).
\end{abstract}

Keywords: NAS, iPLS, valsartan, parameters of merit, chemometrics

\section{Introduction}

Due to the higher stability and convenience, most of medicines are marketed in solid dosages forms. ${ }^{1,2}$ An active pharmaceutical ingredient (API) can be classified as crystalline (crystals, polymorphs, solvates, cocrystals, etc.) or amorphous (non-crystalline). ${ }^{2-4}$ Amorphous solids present some desirable characteristics such as higher solubility, better dissolution profile and compressibility when compared to their crystalline forms. Despite these advantages, amorphous solids usually have higher hygroscopicity and lower chemical and physical stability when compared to the crystalline forms. Amorphous solids tend to crystallize because crystals are thermodynamically more stable. Such transition is a problem since it can affect the bioavailability promoting changes in the dissolution profile. ${ }^{3,5}$

Valsartan is an antihypertensive API ${ }^{6}$ classified as

*e-mail: marcofarias@ufam.edu.br

Editor handled this article: Eduardo Carasek class II (low water solubility) at the biopharmaceutical classification system (BCS). ${ }^{7}$ As valsartan presents low aqueous solubility, the marketed product uses the amorphous solid of this API aiming to reach a better dissolution profile. The amorphous valsartan (VAL-AM) is more propense to degradation than its crystalline forms, thus, special cares are necessary during the manipulation and storage. ${ }^{6}$ The first valsartan medicine was registered at the Food and Drug Administration (FDA) in $1996,{ }^{8}$ thus, after the patent expiration, the generic medicines companies could market products of valsartan. Aiming to have a market protection, the former patent holder deposited a patent for the most thermodynamically stable polymorph. ${ }^{8,9}$ The production of medicines using amorphous APIs is surrounded by the risk of occurrence of crystalline transition, that consists in the crystallization of the amorphous API. So, the characteristics of VAL-AM allows its transition to the protected form (by patent), introducing some legal issues for the generic companies. Therefore, there is a demand to develop analytical methods at the solid state which 
are capable to detect such transformations, since it is not possible to use any method which makes use of dissolution of the sample, such as chromatographic techniques. FDA ${ }^{10}$ proposes a group of analytical techniques to deal with such problems that includes powder X-ray diffraction (PXRD), nuclear magnetic resonance (NMR), differential scanning calorimetry (DSC), thermogravimetry (TGA), among others, however, spectroscopic techniques do not appear among them. As presented in the literature, ${ }^{11}$ Raman and infrared spectroscopies are fully capable of differentiating polymorphs and the alliance of spectroscopic techniques with chemometrics turns these techniques very useful.

The multivariate methods arise to overcome the limitation of univariate methods, namely the quantification in the presence of interferents. Multivariate calibration methods consider that a group of variables give more reliable results than only one variable, ${ }^{12}$ especially when the inverse mode of calibration is used. ${ }^{13}$ One of the drawbacks of the multivariate calibration methods is the complexity of the establishment of the parameters of merit (POM), which turn possible to analyze the quality of the model. Net analyte signal (NAS) is the most used method to calculate the POM. The NAS concept was initially proposed by Lorber, ${ }^{12}$ although Morgan ${ }^{14}$ had discussed a similar concept some years before. According to Olivieri et al. ${ }^{13}$ the NAS is an intrinsic concept in the multivariate calibration because a spectrum may have contribution from various substances in a complex sample, but only a portion of the total signal is due to the contribution of the analyte of interest. Hence, the decomposition of the spectrum into two orthogonal parts can work as a filter showing the part that is uniquely assigned to the analyte of interest. ${ }^{12,13,15}$ The POM for multivariate calibration models can be calculated using the NAS concept giving rise to five parameters: sensitivity (SEN), selectivity (SEL), analytical sensitivity $(\gamma)$, limit of detection (LOD) and limit of quantification (LOQ). Detailed explanations of these and other POM can be found in the literature. ${ }^{13,15}$

The objective of this work was to develop an analytical method to simultaneously quantify crystalline and amorphous valsartan by Raman spectroscopy in the presence of excipients. For that, a binary mixture design was used to prepare samples along the analytical range, and the interval partial least squares (iPLS) was used for calibration. NAS was employed to obtain the POM of the proposed method.

\section{Experimental}

Amorphous valsartan was acquired from Afine Chemical Limited (Sandun, Hangzhou, China) and was used to obtain valsartan form $\mathrm{E}$, as described by Wang et al. ${ }^{6}$ The excipients crospovidone, silicon dioxide and magnesium stearate were acquired in a local drug store (São Carlos, Brazil). The samples were prepared following a binary mixture design of amorphous and crystalline (form E) valsartan, according to Table 1 . It was prepared eleven samples for calibration and five for external validation. Crospovidone (15 mg), magnesium stearate $(15 \mathrm{mg}$ ) and silicon dioxide $(2.5 \mathrm{mg})$ were added to each sample at constant concentration. ${ }^{16}$ The samples were homogenized for $10 \mathrm{~min}$ in a mortar.

Table 1. Concentration of amorphous and crystalline (form E) valsartan in the samples

\begin{tabular}{|c|c|c|c|}
\hline & \multirow{2}{*}{ Sample } & \multicolumn{2}{|c|}{ Valsartan } \\
\hline & & Amorphous / mg & Form E / mg \\
\hline \multirow{11}{*}{ Calibration set } & 1 & 40.40 & 0.00 \\
\hline & 2 & 36.06 & 4.54 \\
\hline & 3 & 32.06 & 8.48 \\
\hline & 4 & 28.26 & 12.16 \\
\hline & 5 & 24.42 & 15.94 \\
\hline & 6 & 20.16 & 20.16 \\
\hline & 7 & 16.14 & 24.28 \\
\hline & 8 & 12.44 & 28.20 \\
\hline & 9 & 8.04 & 32.48 \\
\hline & 10 & 4.60 & 35.96 \\
\hline & 11 & 0.00 & 40.30 \\
\hline \multirow{5}{*}{ Validation set } & 12 & 6.10 & 34.28 \\
\hline & 13 & 14.00 & 25.82 \\
\hline & 14 & 18.02 & 21.98 \\
\hline & 15 & 21.78 & 17.96 \\
\hline & 16 & 34.20 & 6.20 \\
\hline
\end{tabular}

The amorphous and crystalline APIs were characterized by PXRD, TGA and DSC. The PXRD analyses were performed in a Panalytical diffractometer (Massachussets, USA), model Empyream, using a copper source (wavelength of $0.154 \mathrm{~nm}$ ), voltage of $40 \mathrm{kV}$ and emission current of $30 \mathrm{~mA}$. The samples were analyzed from 5 to $45^{\circ}(2 \theta)$ at a scan rate of $0.2^{\circ} \mathrm{min}^{-1}$. Thermal analyses were performed in a SDT Q600 TA Instrument (Delaware, USA) using aluminium crucibles without cover. The parameters were heating rate of $10^{\circ} \mathrm{C} \mathrm{min}^{-1}$ from 10 to $500^{\circ} \mathrm{C}$ under nitrogen gas flow of $30 \mathrm{~mL} \mathrm{~min}^{-1}$.

Raman spectra were acquired using a B\&W Tek i-Raman, model BWS 415-785 (Newark, NJ, USA) with laser at $785 \mathrm{~nm}$ and spectral resolution of $3.5 \mathrm{~cm}^{-1}$. The laser was coupled to a microscope B\&W Tek BAC 151 using a $40 \times$ objective. The spectral range was 110 to $1100 \mathrm{~cm}^{-1}$ and $60 \mathrm{~s}$ of acquisition time. The laser power was adjusted to obtain the best signal to noise ratio for each sample, varying from 50 to $80 \mathrm{~mW}$ (spectra were normalized to eliminate 
differences in the intensities). Ten spectra were collected from each sample at different positions in order to avoid subsampling problems due to the microheterogeneity. ${ }^{17,18}$ An average spectrum was calculated using the ten replicates for each sample.

PLS Toolbox 8.2 from Eigenvector and Matlab version R2017a (license number 40565580) ${ }^{19}$ were used for data processing and modelling. The spectra were normalized by the area to avoid problems related to the variation of the scattering intensity, caused by the difference of size at the particles. ${ }^{20}$ Interval partial least squares was performed using the average spectra, first derivative (Savitzky-Golay) and standard normal variate (SNV). ${ }^{21,22}$ Cross-validation was employed to assess the ideal number of latent variables followed by the calculation of the root mean square errors for calibration (RMSEC), cross-validation (RMSECV) and validation (RMSEV). The model was also evaluated using the NAS concept to calculate the parameters of merit sensitivity, selectivity, analytical sensitivity, limit of detection and limit of quantification.

\section{Results and Discussion}

The absence of peaks in the diffractogram (Figure 1a) confirms the amorphous nature of the valsartan, as described by Rukhaman et $a l .{ }^{23}$ Figure $1 \mathrm{~b}$ shows the peaks for the crystalline valsartan (form E) obtained by recrystallization, showing the main peaks at 9.40, 10.84, 11.62, 14.02, 17.82, 18.76, 26.58 and $31.282 \theta^{\circ}$, as described by Wang et al. ${ }^{6}$

The DSC of amorphous valsartan at Figure $2 \mathrm{c}$ presents two endothermic events, the first one ranging from 68 to $90{ }^{\circ} \mathrm{C}$, probably due to the loss of residual solvent or water, what can be confirmed by the mass loss in TGA at same temperature, in Figure 2a. VAL-AM has a higher tendence for adsorption when compared to VAL-E. ${ }^{6,24,25}$ The second event in Figure $2 \mathrm{c}$ occurs around $99{ }^{\circ} \mathrm{C}$ and does not involve mass loss, according TGA (Figure 2a). This event could be related to the melting of a metastable crystal formed during the heating, since it presents a sharp endothermic peak,

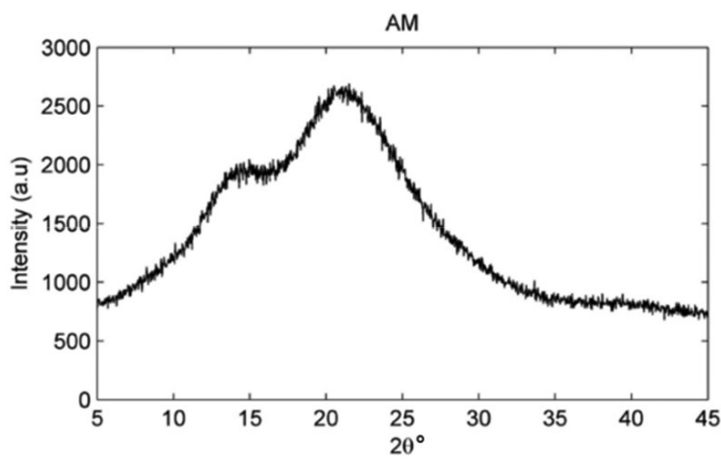

Figure 1. PXRD of amorphous (AM) and crystalline (E) valsartan. however, it was not observed any significant exothermic peak indicating a previous crystallization. The TGA of VAL-AM (Figure 2a) and VAL-E (Figure 2b) are very similar after $100{ }^{\circ} \mathrm{C}$, and the mass loss events at 210 and $400{ }^{\circ} \mathrm{C}$, for both solids, are related to the decomposition of the API.

The DSC curve for the crystalline form $\mathrm{E}$ of valsartan (Figure 2d) presents one endothermic peak at $136{ }^{\circ} \mathrm{C}$ corresponding to the melting point. ${ }^{6}$

The Raman spectra of excipients and valsartan are present in the Figure 3. There are relevant differences between the spectra of VAL-AM and VAL-E. These differences are related to the fact that the VAL-AM does not have a well-ordered crystalline arrangement and has a distinct chemical environment. The differences between the spectra of VAL-AM and VAL-E generate a unique pattern for each form. ${ }^{26,27}$ In addition, Raman spectroscopy also gives information about the crystalline pattern due to the vibration of the crystalline structure, what turns Raman spectroscopy a special tool at the recognition of polymorphism. ${ }^{28}$

Figure 4 presents the normalized average spectra of calibration samples. Despite the clear differences among the spectra of VAL-AM and VAL-E, the multivariate analyses is necessary since it allows the identification of very small changes in the spectra, which are related to the physical state of VAL. ${ }^{28}$

A regression model based on partial least squares by interval (iPLS) was built to quantify the VAL-AM and VAL-E in the samples. iPLS creates models using different intervals of the spectra in order to find the intervals which produce the better models. Cross-validation was employed to find the ideal number of latent variables (LV's), as presented in Figure 5. At least two latent variables were needed to obtain lower prediction errors. Two latent variables were enough to explain 99.81 and $99.54 \%$ of variance in the models for VAL-AM and VAL-E, respectively.

The iPLS model was built using two LV's and interval windows of 20 variables. Figure 6 shows the selected intervals for the best model: 201-220, 721-740 and

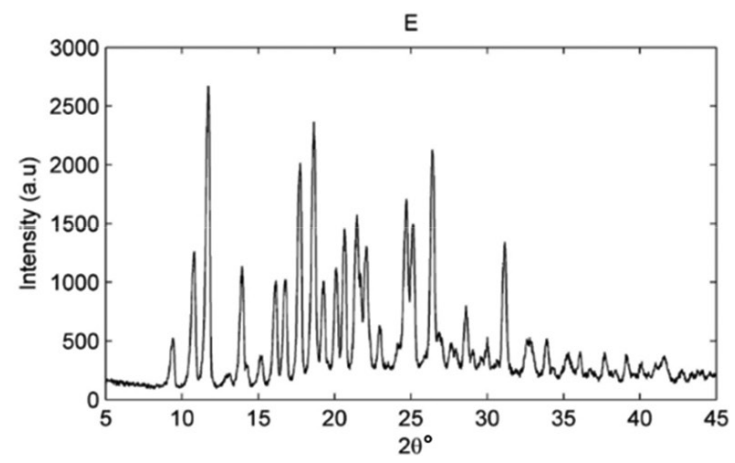



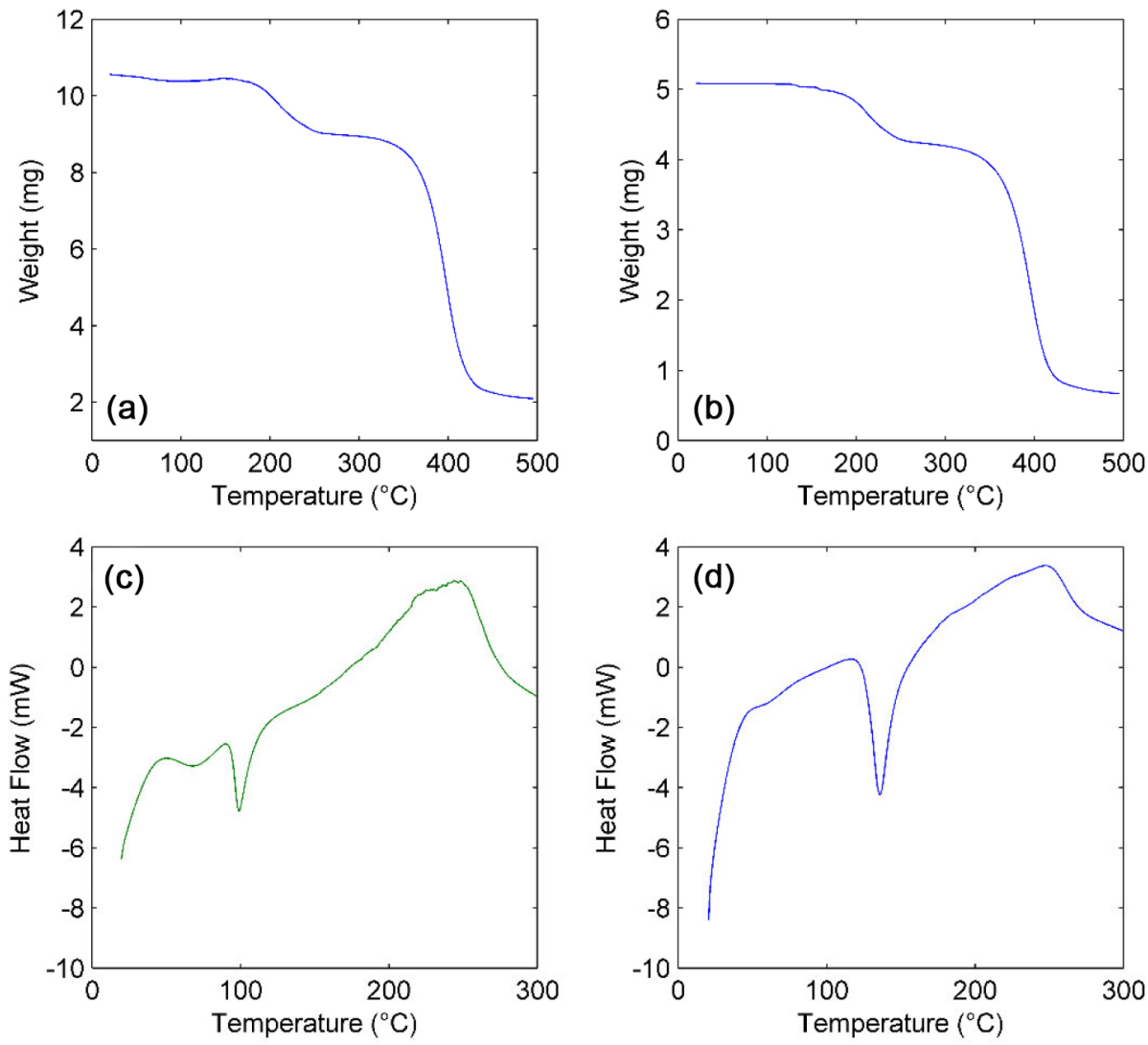

Figure 2. (a) TGA of VAL-AM; (b) TGA of VAL-E; (c) DSC of VAL-M and (d) DSC of VAL-E.

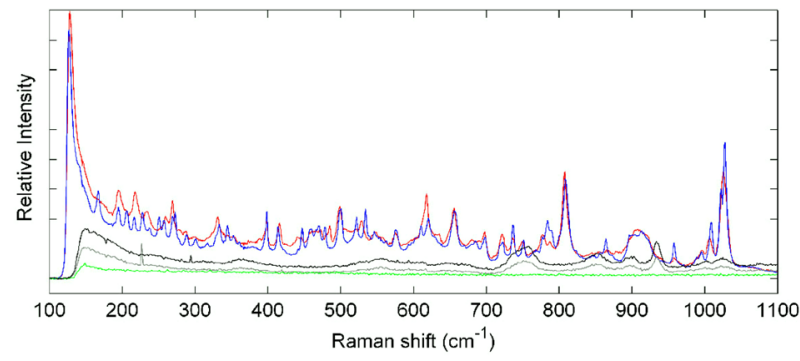

Figure 3. Raman spectra of crospovidone (green), magnesium stearate (grey), colloidal silicon dioxide (black), VAL-E form (blue) and VAL-AM (red).

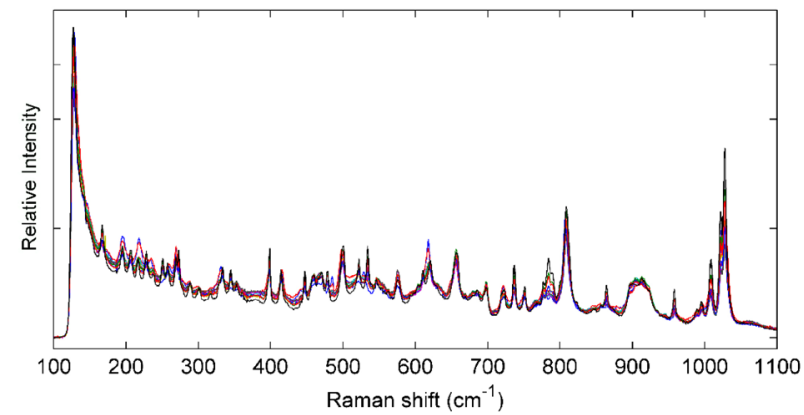

Figure 4. Spectra of calibration set.

1001-1020 $\mathrm{cm}^{-1}$. RMSEC, RMSECV, RMSEV, coefficient of determination $\left(\mathrm{R}^{2}\right)$, SEL, SEN, $\gamma, \mathrm{LOD}$ and LOQ values are present at Table 2 .

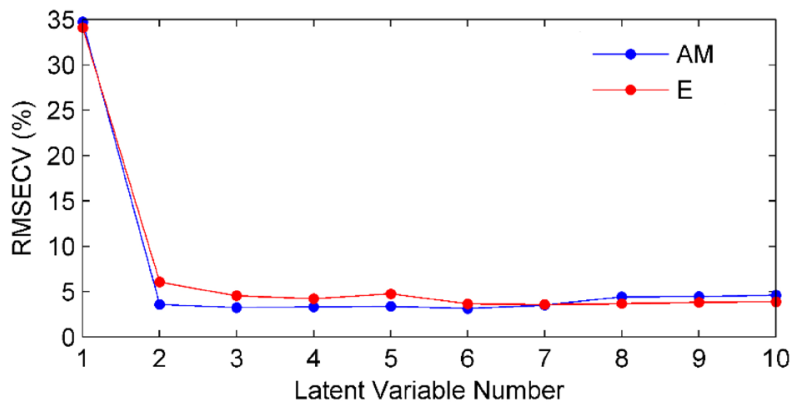

Figure 5. RMSECV values versus number of latent variables.

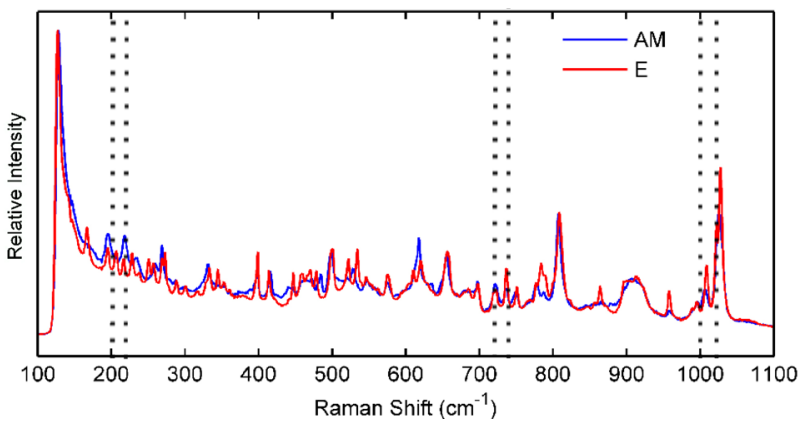

Figure 6. Intervals used in the iPLS model.

The POM, expressed at the Table 2, shows a predictive capacity of the model, with RMSECV and RMSEV values ranging from 3.58 to $6.05 \%$. Despite these errors seems 
Table 2. Parameters and the parameters of merit (POM) of the multivariate calibration model

\begin{tabular}{cccc}
\hline \multirow{2}{*}{ POM } & \multicolumn{2}{c}{ Polymorph } \\
\cline { 3 - 4 } & & VAL-AM & VAL-E \\
\hline \multirow{4}{*}{ Accuracy } & RMSEC / $\%, \mathrm{~m} / \mathrm{m})$ & 3.02 & 4.78 \\
& $\mathrm{RMSECV} /(\%, \mathrm{~m} / \mathrm{m})$ & 3.58 & 6.05 \\
& $\mathrm{RMSEV} /(\%, \mathrm{~m} / \mathrm{m})$ & 3.89 & 4.32 \\
& $\mathrm{R}^{2}$ & 0.99 & 0.97 \\
\hline \multirow{6}{*}{ Precision } & $\mathrm{SEN}$ & 0.1243 & 0.1367 \\
& $\mathrm{SEL}$ & 0.61 & 0.69 \\
& $\gamma / \%-1$ & 600 & 633 \\
& $\gamma^{-1} / \%$ & $1.67 \times 10^{-3}$ & $1.58 \times 10^{-3}$ \\
& LOD / \% & 2.89 & 2.63 \\
& LOQ / \% & 8.80 & 7.97 \\
\hline
\end{tabular}

VAL-AM: valsartan amorphous; VAL-E: valsartan crystalline; RMSEC: root mean square error of calibration; RMSECV: root mean square error for cross validation; RMSEV: root mean square error validation; SEN: sensitivity; SEL: selectivity; $\gamma$ : analytical sensitivity; $\gamma^{-1}$ : inverse analytical sensitivity; LOD: limit of detection; LOQ: limit of quantification; $\mathrm{R}^{2}$ : coefficient of determination.

high for an analytical method, it is necessary to understand that the quantification is about two different physical states of the same molecule. The $\mathrm{R}^{2}$ related to the real versus predicted values were 0.99 for VAL-AM, and 0.97 for VAL-E. The graphic of these correlations is presented at Figure 7.

The RMSE values do not show the whole picture of the model, showing only a trend and the accuracy. Considering the way these parameters are calculated, the values are related to a mean value for all individual samples, in this way, it is important to evaluate the POM. The level of instrumental noise at the measures is used to calculate the POM. The average noise was $1.09 \times 10^{-3}$ signal units, which was obtained using 5 measures at a blank sample, considering the spectral ranges $721-740$ and $1001-1020 \mathrm{~cm}^{-1}$.

SEN is the variance of the instrumental signal related to concentration of the analyte. ${ }^{11,13,29}$ The model is slightly more sensitive to VAL-E than to VAL-AM, what was

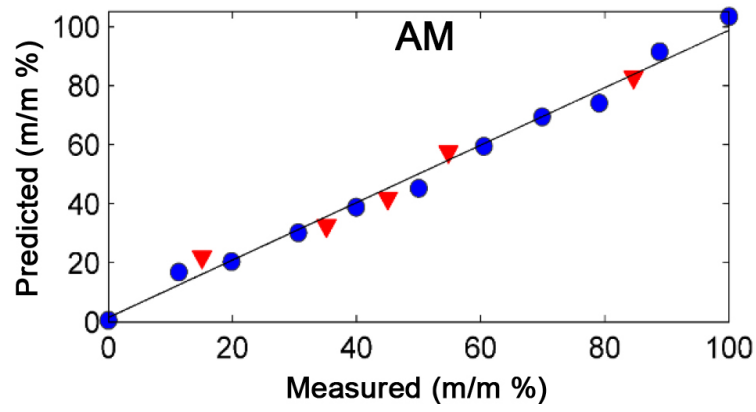

expected since the Raman spectrum of VAL-E was more intense. The model presents a sensitivity that seems low, but it is worthy to point that the values of SEN are related to the normalized spectra with a maximum signal nearly to 0.14 (Figure 4), and the instrumental noise is around $1.09 \times 10^{-3}$. Therefore, the SEN is two orders of magnitude higher than the instrumental noise.

According to the values of SEL, described at the Table 2, the model is slightly more selective for VAL-E. The SEL can be easily understood considering that a model is completely selective for an analyte if SEL $=1$ and completely nonselective when SEL $=0$.

The analytical sensitivity $(\gamma)$ is obtained by the division of sensitivity by instrumental noise. Considering that the Raman intensity varies linearly with the content of analytes, it is possible to consider that $\gamma$ is a measure of how much of the signal is related to the analyte concentration changes. The inverse of analytical sensitivity $\left(\gamma^{-1}\right)$ is physically more comprehensible, because it shows how much in mass proportion among polymorphs and excipients $(\% \mathrm{~m} / \mathrm{m})$ are necessary to cause a change of one unit at the signal intensity. As presented at Table 2, it is necessary $1.67 \times 10^{-3} \%$ in mass of VAL-AM (nearly $0.07 \mathrm{mg}$, considering $40 \mathrm{mg}$ of the API) to promote a change of one unit at the signal intensity, and $1.58 \times 10^{-3} \%$ in mass $(0.06 \mathrm{mg})$ of VAL-E to promote the same change at the signal intensity, showing that the model is slightly more sensitive for VAL-E .

Finally, the LOD shows how much of each solid phase $(\mathrm{m} / \mathrm{m})$ is discernible from the instrumental noise. VAL-E presents a higher sensitivity and a LOD of $2.63 \times 10^{-2}(\mathrm{~m} / \mathrm{m})$, it means that this polymorph is detectable if its content in the samples ( $40 \mathrm{mg}$ of API) is higher than $1.05 \mathrm{mg}$. For VAL-AM the LOD is $2.89 \times 10^{-2}(\mathrm{~m} / \mathrm{m})$ and is necessary an amount of $1.16 \mathrm{mg}$ to detect the amorphous phase in a sample of $40 \mathrm{mg}$ of API. For the LOQ, the minimal mass that could be quantified was $3.52 \mathrm{mg}$ for VAL-AM and $3.19 \mathrm{mg}$ for VAL-E. All samples used in the calibration or validation set had an API content higher than the LOQ and LOD.

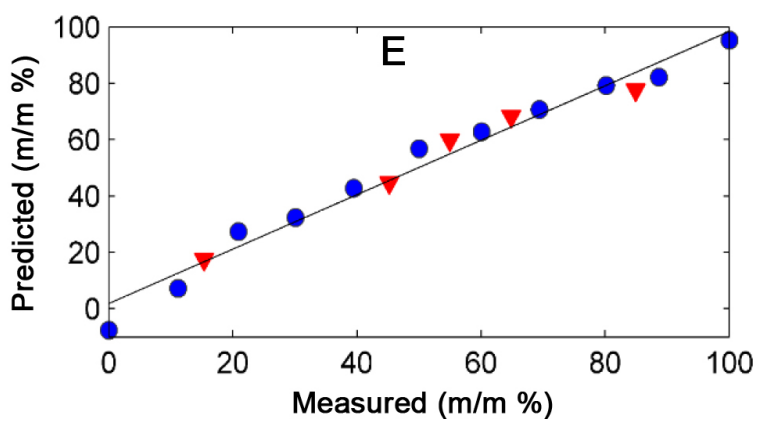

Figure 7. Real vs. predict values for VAL-AM and VAL-E. Blue circles and red triangles are calibration and validation samples, respectively. 


\section{Conclusions}

The method presented in this paper was able to simultaneously quantify mixtures of VAL-AM and VAL-E in the presence of excipients using Raman spectroscopy allied to multivariate analysis. The method can be useful for quality control in pharmaceutical companies since valsartan is marketed as an amorphous API, which is susceptible to crystallization, what can change the dissolution and bioavailability. The usual parameters of multivariate methods (RMSECV, RMSEV, RMSEC) and others POM like SEN, SEL, $\gamma, \gamma^{-1}$ and LOD, calculated using the NAS approach, demonstrated that the model presents prediction power to quantify the content of VAL-AM and VAL-E in the presence of excipients.

\section{Acknowledgments}

The authors are grateful to: LabMat (Laboratório de Materiais), for the PXRD analyses; and Conselho Nacional de Desenvolvimento Científico e Tecnológico (CNPq); Coordenação de Aperfeiçoamento de Pessoal de Nível Superior (CAPES); Financiadora de Estudos e Projetos (FINEP); Fundação de Amparo à Pesquisa do Estado do Amazonas (FAPEAM); Ministério de Ciência, Tecnologia, Inovações e Comunicação (MCTIC) and Universidade Federal do Amazonas (UFAM) for the financial support and fellowship.

\section{References}

1. Hilfiker, R.; von Raumer, M.; Polymorphism in the Pharmaceutical Industry, $5^{\text {th }}$ ed.; Hilfiker, R.; von Raumer, M., eds.; Wiley-VCH Verlag GmbH \& Co. KGaA: Weinheim, Germany, 2018.

2. Stahly, G. P.; Cryst. Growth Des. 2007, 7, 1007.

3. Gupta, P. K.; J. Non-Cryst. Solids 1996, 195, 158.

4. Morissette, S. L.; Almarsson, Ö.; Peterson, M. L.; Remenar, J. F.; Read, M. J.; Lemmo, A. V.; Ellis, S.; Cima, M. J.; Gardner, C. R.; Adv. Drug Delivery Rev. 2004, 56, 275.

5. Yu, L.; Adv. Drug Delivery Rev. 2001, 48, 27.

6. Wang, J.; Wang, X.; Lu, L.; Mei, X.; Cryst. Growth Des. 2013, 13, 3261 .

7. Food and Drug Administration (FDA), The Biopharmaceutics Classification System (BCS) Guidance, https://www.fda. gov/about-fda/center-drug-evaluation-and-research-cder/ biopharmaceutics-classification-system-bcs-guidance, accessed in September 2021.
8. Food and Drug Administration (FDA), https://www. accessdata.fda.gov/scripts/cder/daf/index.cfm?event=overview. process\&ApplNo=021283, accessed in September 2021.

9. Burgbacher, J.; Hahn, B. T.; Rampf, F. A.; Schneeberger, R.; WO pat. 2012/016969, 2012 (CA 2806657).

10. Food and Drug Administration (FDA) U. S.; Guidance for Industry ANDAs: Pharmaceutical Solid Polymorphism Chemistry, Manufacturing, and Controls Information; FDA: Rockville, 2007, p. 264-269.

11. Palermo, R. N.; Short, S. M.; Anderson, C. A.; Tian, H.; Drennen III, J. K.; J. Pharm. Innovation 2012, 7, 56.

12. Lorber, A.; Anal. Chem. 1986, 58, 1167.

13. Olivieri, A. C.; Chem. Rev. 2014, 114, 5358.

14. Morgan, D. R.; Appl. Spectrosc. 1977, 31, 404.

15. Olivieri, A. C.; Faber, N. M.; Ferré, J.; Boqué, R.; Kalivas, J. H.; Mark, H.; Pure Appl. Chem. 2006, 78, 633.

16. Handbook of Pharmaceutical Excipients, $6^{\text {th }}$ ed.; Rowe, R. C.; Sheskey, P. J.; Quinn, M. E., eds.; Pharmaceutical Press: London and Chicago, 2009.

17. Bell, S. E. J.; Beattie, J. R.; McGarvey, J. J.; Peters, K. L.; Sirimuthu, N. M. S.; Speers, S. J.; J. Raman Spectrosc. 2004, 35,409 .

18. Griffen, J. A.; Owen, A. W.; Andrews, D.; Matousek, P.; Spectroscopy 2017, 32, 37.

19. PLS Toolbox, 8.2; Eigenvector Research Inc., MathWorks, USA, 2017.

20. Gemperline, P.; Practical Guide to Chemometrics, $2^{\text {nd }}$ ed.; CRC Press: Boca Raton, FL, 2006.

21. Savitzky, A.; Golay, M. J. E.; Anal. Chem. 1964, 36, 1627.

22. Barnes, R. J.; Dhanoa, M. S.; Lister, S. J.; Appl. Spectrosc. 1989, 43, 772 .

23. Rukhaman, I.; Flyaks, E.; Koltai, T.; Aronhime, J.; US Patent 7,105,557 B2 2006, 37.

24. Skotnicki, M.; Gaweł, A.; Cebe, P.; Pyda, M.; Drug Dev. Ind. Pharm. 2013, 39, 1508.

25. Júlio, T. A.; Zâmara, I. F.; Garcia, J. S.; Trevisan, M. G.; Braz. J. Pharm. Sci. 2013, 49, 645.

26. D’Ippolito, V.; Andreozzi, G. B.; Bersani, D.; Lottici, P. P.; J. Raman Spectrosc. 2015, 46, 1255.

27. Pankin, D.; Kolesnikov, I.; Vasileva, A.; Pilip, A.; Zigel, V.; Manshina, A.; Spectrochim. Acta, Part A 2018, 204, 158.

28. Hédoux, A.; Adv. Drug Delivery Rev. 2016, 100, 133.

29. Lorber, A.; Faber, K.; Kowalski, B. R.; Anal. Chem. 1997, 69, 1620.

Submitted: June 13, 2021

Published online: September 20, 2021 